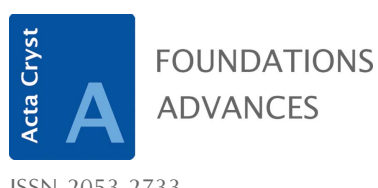

ISSN 2053-2733
Keywords: book review; quantum physics; statistical physics.

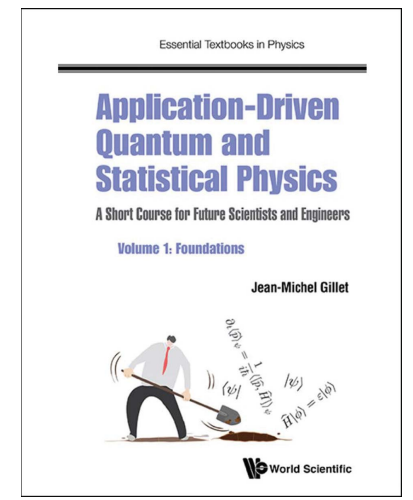

(C) 2020 International Union of Crystallography

\section{Application-Driven Quantum and Statistical Physics. A Short Course for Future Scientists and Engineers. Volume 1: Foundations. Volume 2: Equilibrium. By Jean-Michel Gillet. World Scientific, 2018. Hardcover, price GBP 86.00 each. Volume 1: pp. 300, ISBN 978-1-78634-554-7. Volume 2: pp. 336, ISBN 978-1-78634-557-8.}

\author{
Karlheinz Schwarz*
}

Institute of Materials Chemistry, Vienna University of Technology, Getreidemarkt 9/165-TC, Vienna, A-1060, Austria. *Correspondence e-mail: kschwarz@theochem.tuwien.ac.at

In these two books we learn about several aspects of classical physics and the reasons for introducing quantum physics. In order to find solutions of the corresponding problems one often makes idealizations, which deviate from realistic cases but allow one to solve them exactly (for model systems). Many of these aspects should be kept in mind by the reader. The author puts the main emphasis on mathematical physics, which might be a challenge for the reader.

In the first book (Foundations), we find a general introduction starting at the beginning of the 20th century. This was the time when classical physics could not explain some experimental results and thus quantum physics was born. New physical constants were introduced, such as $h$, the Planck constant, and new rules that are relevant at the microscopic scale. One speciality of this book is that biographical notes for some key actors are included. Modern textbooks of physics often ignore these historical details. We can easily see which topics of the new physics the author described in the book by listing the names of these actors (most of them Nobel laureates): Kelvin, Planck, von Lenard, Hertz, Frank, Einstein, Compton, Bohr, de Broglie, Davisson, Germer, Born, Heisenberg, Schrödinger, Dirac, Lagrange, Ehrenfest, Feynman, and even the more recent Nobel laureates Rohrer and Binning.

The book begins by explaining the new concepts in physics, which nowadays are well known: from waves to particles, blackbody radiation, photons, the photoelectric effect, Compton scattering or when conventional particles behave like waves. Next, it illustrates how Bohr's theory of the transition between atomic levels can be helpful. The author takes an unusual case, namely analysing a portrait of Goya, to illustrate the importance of atomic levels.

The experimental result, that particles exhibit wave-like behaviour under particular circumstances, led to the development of wavefunction schemes introduced by Schrödinger. This was the start of the dual wave and particle description and Heisenberg's inequalities, which is the intrinsic impossibility of a particle having simultaneously a fixed value for its position and its momentum (or energy and lifetime). It is not surprising that this book presents an extensive mathematical treatment in order to lay the groundwork for a fundamental understanding. Concepts like wavefunction, probabilities, stationary state and operators require a mathematical definition.

For idealized cases, e.g. piecewise constant potentials, the quantum behaviour can be studied and feasible solutions made. Such a procedure provides first results, which are important before one starts to develop a more realistic treatment of real systems. These idealized case studies are useful for systems like quantum dots or scanning tunnelling microscopy and others as presented in this section.

The author calls the next chapter 'mathematical artillery', which is a good description. This chapter defines concepts like a state vector $|\psi(t)\rangle$ called a ket. It would have been nice to mention that this name comes from bracket spit into a bra and ket vector. Also 
described are topics like Hilbert space, the superposition principle, observables, eigenvalues, (Hermitian) operators and the time-dependent Schrödinger equation. In this chapter, the author describes how these concepts become important in applications, like quantum cryptography, or in describing the flipping of the ammonia molecule.

The last part of Volume 1 addresses the relation between the classical and quantum physics and goes from the 'least action principle' or from Lagrange to the Hamilton. In addition, the reader can find out about Ehrenfest's theorem or Bohr's correspondence principle.

All chapters contain questions (and answers provided later on), which is a helpful tool. When the author describes experiments related to various topics, several details are nicely presented in figures. The formalism is often rather mathematical and thus a challenge for the reader.

The second book, called Equilibrium, starts with model Hamiltonians and approximations. The harmonic oscillator is a good illustration of how to go from classical physics to a quantum-mechanical treatment. For the latter, two different mathematical schemes are presented, namely the use of creation and annihilation operators and solving the corresponding differential equation, for which an asymptotic solution is combined with an infinite series. However, to satisfy the normalization condition of this wavefunction, one must truncate the infinite series to a polynomial leading to the quantum number and the famous zero-point energy. Even at this lowest energy, the position is not fixed, satisfying Heisenberg's uncertainty principle. The author illustrates these fundamental principles for several applications, like Mössbauer or vibrational spectroscopy, and goes on to present cases of how to detect explosives. We start with a mathematical derivation of general rules followed by the interpretation of results looking at applications, for example van der Waals interactions.

After reading about the effects of translation, we find details of the rotation starting with the idealized case of a rigid rotator. For this the rules for the angular momentum operator are derived and the quantum states with the related spherical harmonics are defined and illustrated graphically (showing their symmetries). The addition of angular momenta is another important aspect for real systems. For this part, the $\mathrm{CO}$ molecule is the chosen application.

An orbital angular momentum of a charged particle generates a magnetic moment. The famous Stern-Gerlach experiment initiated the introduction of the spin model following the idea of Uhlenbeck and Goudsmit's hypothesis. In short, an electron has a new angular momentum with the quantum number $\frac{1}{2}$ with only two possible spin states and thus an additional degree of freedom. Next we find how the Pauli matrices are defined. This is the basis for the Pauli principle for the wavefunction of a system of $N$ indistinguishable fermions. The author presents the concept of fermions and bosons in connection with nuclear physics.

The next section, called central Coulombic potential, describes how to solve hydrogenic systems. One can separate the full Schrödinger equation according to the Born-
Oppenheimer approximation, since the nuclear and electronic motion are essentially independent. The electronic part (called the central field problem) is solved by representing the wavefunction as a radial function times spherical harmonics. The radial function is a combination of the asymptotic solutions (for small and large distances) and an associated Laguerre polynomial. This one-electron model still needs some fine structure corrections including relativistic effects, like spin-orbit coupling.

Based on the previous section we find the treatment of the $N$-electron atom. One solves this very important problem approximately using the variational principle. The author mentions that the latter is used in most ' $a b$ initio' methods to retrieve quantum properties from first principles without resort to empirical parameters. He presents many details, like mean field, Hartree product, Pauli principle and Slater determinant. The results allow a fundamental understanding of the periodic table of the elements, introduced by Mendeleev 150 years ago. Quantum mechanics made it possible to explain the periodic table using the quantum numbers derived from the hydrogen atom and then explain the electronic structure of atoms with their shell structure (following the aufbau principle). However, a variety of approximations had to be introduced in order to justify the periodic table.

The next section of the book gives a short summary of 'statistical physics' for treating macroscopic systems, from gases and liquids to solids. The main rules are known under the term thermodynamics. For this chapter the author assumes that the reader knows the main concepts, but he briefly summarizes them, for example for the four laws. In addition, the author describes various topics such as the internal energy for isolated systems, an adiabatic process, reversible versus irreversible transitions, enthalpy and entropy, the Gibbs's free energy, and the equation of state. Such concepts are relevant for phase transitions, Maxwell's relation or the response function. We can read how one can apply these concepts for a magnetic field or chemical reactions.

The macroscopic observation emerges from an averaging of microscopic possibilities, based on quantum and classical physics. The ergodic principle is the equivalence between time average and ensemble average. Another topic is Shannon's entropy. For an isolated system, a microcanonical ensemble represents all accessible microstates. The thermodynamic potentials and the partition function (Zustandssumme) play a key role in statistical physics as demonstrated for the well known Haber-Bosch process for the synthesis of ammonia.

In these two books the author combines several important topics from classical and quantum physics to statistical physics and thermodynamics. For readers knowing the basics they can gain new insight by understanding mathematical rules for physical properties or learning the historic developments and the relevance for modern applications. The questions and answers provided can be helpful in this learning process. These books are not easy to read, having an unconventional presentation, but are recommended to graduate students of theoretical physics. 